\title{
Coma recovery scale-r: variability in the disorder of consciousness
}

\author{
MD. Cortese ${ }^{1}$, F. Riganello' , F. Arcuri ${ }^{1}$, ME. Pugliese ${ }^{1}$, LF. Lucca' ${ }^{1}$ G. Dolce ${ }^{1}$ and WG. Sannita ${ }^{2,3^{*}}$
}

\begin{abstract}
Background: Despite evidence from neuroimaging research, diagnosis and early prognosis in the vegetative (VS/UWS) and minimally conscious (MCS) states still depend on the observation of clinical signs of responsiveness. Multiple testing has documented a systematic variability during the day in the incidence of established signs of responsiveness. Spontaneous fluctuations of the Coma Recovery Scale-revised (CRS-r) scores are conceivable.

Methods: We retrospectively analyzed the CRS-r repeatedly administered to 7 VS/UWS and 12 MCS subjects undergoing systematic observation during a conventional 13 weeks. rehabilitation plan.

Results: The CRS-r global, visual and auditory scores were found higher in the morning than at the afternoon administration in both VS/UWS and MCS subgroups over the entire period of observation. The probability for a VS/UWS subject of being classified as MCS at the morning testing at least once during the 13 weeks. observation was as high as $30 \%$, i.e., compatible with the reported misdiagnosis rate between the two clinical conditions.

Conclusions: Multiple CRS-r testing is advisable to minimize the risk of misclassification; estimates of spontaneous variability could be used to characterize with greater accuracy patients with disorder of consciousness and possibly help optimize the rehabilitation plan.
\end{abstract}

Keywords: CRS-r, Disorders of consciousness, Variability, Vegetative state, Minimally conscious state

\section{Background}

Diagnosis and the early prognosis of subjects in the vegetative state/unresponsive wakefulness syndrome (VS/ UWS) [1, 2] or minimally conscious state (MCS) [3-7] still depend on the clinical evaluation of responsiveness $[4,5,8]$, while functional assessment by neuroimaging remains mostly limited to research [5, 9-15]. The diagnostic error between the VS/UWS and MCS reportedly still hovers up to $25-45 \%$, with possible implications in the patients' prognosis, treatment, allocation of resources, etc. $[3,4,16]$. Clinical scales have been developed to provide the attending physician and therapist with reliable criteria of behavioral assessment and to allow more accurate evaluations [3, 16, 17]. Among these, the Coma Recovery Scale - revised (CRS-r) is an established and widely used tool $[18,19]$, with higher

\footnotetext{
*Correspondence: wgs@dism.unige.it

${ }^{2}$ Department of Neuroscience, Ophthalmology and Genetics, University of Genova, 3, Largo P. Daneo, 16132 Genova, Italy

${ }^{3}$ Department of Psychiatry, State University of New York, Stony Brook, NY, USA

Full list of author information is available at the end of the article
}

percentage of MCS subjects correctly diagnosed and better overall classification accuracy than the current clinical criteria [7].

The within/between subject (spontaneous) variability of the signs in use to evaluate brain responsiveness in VS/ UWS and MCS is a possible critical issue adding to the existing controversy on their pathophysiological meaning $[17,20]$. The visual pursuit response, a major CRS-r item recognizable in $70-80 \%$ of subjects in MCS $[9,21,22]$ and key marker of evolution from VS/UWS [23, 24], has been also reported with lower incidence $(20-30 \%)$ in subjects otherwise diagnosed as VS/UWS [20, 25-28]. Multiple testing has documented systematic spontaneous fluctuations in the incidence of this response in the course of the day in both VS/UWS and MCS subjects; the overall chance of observing it at least once per day was $~ 33 \%$ and $\sim 62 \%$ in the VS/UWS and MCS subgroups, respectively, with maxima at 10.30 am and $3.00 \mathrm{pm}$ and no response at postprandial time in both subjects' groups [29]. Individual variability may suggest limited diagnostic accuracy for the visual pursuit response and, by extension, risk 
of erroneous CRS-r classification when subjects are tested at a single time point of the day. Purpose of this study was to analyze the spontaneous fluctuation over time of the CRS-r scores.

\section{Methods}

We studied retrospectively the CRS-r scores obtained from two subgroups of subjects with disorder of consciousness undergoing a standard rehabilitation plan. They had been diagnosed by the attending physician as being in chronic VS/UWS ( $n=7$; age $51.8 \pm 11.6$ years.) or MCS ( $n=12$; age $51.4 \pm 17.4$ years.) at the beginning of treatment; diagnosis was based on the current clinical criteria and guidelines by the Aspen group [19]. The CRS$\mathrm{r}$ global score was equal or lower than 7 (mean $=6.42 \pm$ 0.49 ) in VS/UWS subjects and between 9 and 15 in the MCS patients (mean $=11.08 \pm 1.75)$. Subjects clinically unstable, under treatment with (neuro)active drugs or beta-blockers, with anoxia, history of psychiatric disorder, concurrent systemic diseases, or evidence of recurrent pain were not considered. Subjects requiring Arousal Facilitation Protocol procedures (AFP) were also excluded in order to avoid the interference of these procedures on their responses to the CRS-r stimulus condition. The VS/ UWS and MCS subjects' subgroups did not differ as to age (Mann-Whitney's test: $\mathrm{z}=-0.466, p>0.05$ ) or length of hospitalization (1144.3 \pm 551.2 days in VS/UWS and $1711.4 \pm 455$ days in MCS; $\mathrm{z}=-1945, p>0.05)$. Summary demographics and clinical information are reported in Tables 1 and 2 .

The study has been approved by the local public health care Ethical Committee (Provincial Health Authority of Crotone). The patients' relatives and caregivers were informed about the study aims and gave their consent to the use of the patients' data, which have been always treated under condition of anonymity. The ethical principles of the Declaration of Helsinki (1964) by the World Medical Association concerning human experimentation were followed.

Patients were treated following a standard plan with alternating 2 weeks. periods of intensive rehabilitation (two sessions/day, morning and afternoon; hereafter Phase A) and 1 week. periods of standard rehabilitation (one session/day; Phase B) for a total of 13 weeks. The protocol began and ended with the phase B (Fig. 2). Subjects received nursing care before 9.00 a.m. and after 5.00 p.m. and were fed at noon compliant to the unit schedule; the rehabilitation sessions and CRS-r evaluation always began at least $30 \mathrm{~min}$. after the morning nursing care in order to avoid induced arousal or other possible interference.

CRS-r was always administered before rehabilitation and in the two time windows of the day when responsiveness

Table 1 Summary demographics and clinical information at the beginning of the rehabilitation plan. (The patients' relatives and caregivers gave their consent to the use of the patients' data)

\begin{tabular}{|c|c|c|c|c|c|c|c|}
\hline & \multirow[t]{2}{*}{ Sex } & \multirow{2}{*}{$\begin{array}{l}\text { Age } \\
\text { range }\end{array}$} & \multirow[t]{2}{*}{ Aetiology } & \multicolumn{3}{|l|}{ CRS-r } & \multirow{2}{*}{$\begin{array}{l}\text { Time from } \\
\text { brain injury (days) }\end{array}$} \\
\hline & & & & Total score & Visual subscore & Auditory subscore & \\
\hline \multirow[t]{7}{*}{ VS/UWS } & \multirow[t]{5}{*}{ Female } & \multirow[t]{5}{*}{$38-73$} & Vascular & 6 & 1 & 1 & 662 \\
\hline & & & Other & 7 & 0 & 1 & 2278 \\
\hline & & & Vascular & 6 & 1 & 1 & 663 \\
\hline & & & Vascular & 7 & 0 & 1 & 958 \\
\hline & & & Vascular & 6 & 0 & 2 & 1256 \\
\hline & \multirow[t]{2}{*}{ Male } & \multirow[t]{2}{*}{$41-65$} & Traumatic & 6 & 1 & 1 & 1199 \\
\hline & & & Traumatic & 7 & 1 & 1 & 1201 \\
\hline \multirow[t]{12}{*}{ MCS } & \multirow[t]{7}{*}{ Male } & \multirow[t]{7}{*}{$31-65$} & Vascular & 9 & 3 & 2 & 1199 \\
\hline & & & Traumatic & 10 & 3 & 2 & 1492 \\
\hline & & & Traumatic & 10 & 3 & 0 & 1958 \\
\hline & & & Traumatic & 13 & 3 & 1 & 2380 \\
\hline & & & Traumatic & 11 & 2 & 1 & 2455 \\
\hline & & & Traumatic & 11 & 2 & 1 & 2345 \\
\hline & & & Traumatic & 15 & 3 & 3 & 2399 \\
\hline & \multirow[t]{5}{*}{ Female } & \multirow[t]{5}{*}{$35-79$} & Traumatic & 9 & 3 & 2 & 2251 \\
\hline & & & Vascular & 10 & 3 & 1 & 1393 \\
\hline & & & Vascular & 10 & 3 & 1 & 1773 \\
\hline & & & Vascular & 13 & 2 & 1 & 2298 \\
\hline & & & Traumatic & 12 & 3 & 1 & 1542 \\
\hline
\end{tabular}


Table 2 Summary demographics and clinical information at the beginning of the rehabilitation plan. (The patients' relatives and caregivers gave their consent to the use of the patients' data)

\begin{tabular}{|c|c|c|c|c|c|c|}
\hline Score & Auditory scale & Visual scale & Motor scale & OromotorNerbal scale & Communication scale & Arousal scale \\
\hline 6 & - & - & Functional Object Use & - & - & - \\
\hline 5 & - & Object Recognition & $\begin{array}{l}\text { Automatic Motor } \\
\text { Response }\end{array}$ & - & - & - \\
\hline 4 & $\begin{array}{l}\text { Consistent movement } \\
\text { to command }\end{array}$ & $\begin{array}{l}\text { Object Localization: } \\
\text { Reaching }\end{array}$ & Object Manipulation & - & - & - \\
\hline 3 & $\begin{array}{l}\text { Reproducible Movement } \\
\text { to Command }\end{array}$ & Visual Pursuit & $\begin{array}{l}\text { Localization to Noxious } \\
\text { Stimulation }\end{array}$ & $\begin{array}{l}\text { Intelligible } \\
\text { Verbalization }\end{array}$ & - & Attention \\
\hline 2 & Localization to Sound & Fixation & Flexion Withdrawal & $\begin{array}{l}\text { Vocalization/Oral } \\
\text { Movement }\end{array}$ & Functional: Accurate & $\begin{array}{l}\text { Eye Opening w/o } \\
\text { Stimulation }\end{array}$ \\
\hline 1 & Auditory Startle & Visual Startle & Abnormal Posturing & $\begin{array}{l}\text { Oral Reflexive } \\
\text { Movement }\end{array}$ & $\begin{array}{l}\text { Non-Functional: } \\
\text { Intentional }\end{array}$ & $\begin{array}{l}\text { Eye Opening with } \\
\text { Stimulation }\end{array}$ \\
\hline 0 & None & None & None/Flaccid & None & None & Unarousable \\
\hline
\end{tabular}

had previously proved highest in a previous study (9.30-11.00 a.m.; 3.00-4.00 p.m.) [29]. CRS-r administration was once a week in phases A (in the morning the first week and in the afternoon the second week); in phases $\mathrm{B}$, it was administered in the morning and afternoon at the beginning and end of the week. The CRS-r was administered following the international guidelines by two expert examiners (a neuropsychologist and an occupational therapist) who were requested to examine each patient together and to reach an agreement on each measured item. When tested, patients were comfortably sitting on an armchair in a familiar setting with a constant temperature of $24{ }^{\circ} \mathrm{C}$ in the absence of environmental noise or interference; in no case, patients were reported to have showed discomfort in the presence of the examiners or during testing. Only patients with matching numbers of assessments were considered in the study. Scores from 342 CRS-r administrations were obtained in total (18 times per subject).

The CRS-r global scores and subscores at the morning and afternoon ratings and at the beginning and end of the protocol were compared. Statistical processing was by Wilcoxon signed-rank test, after collapsing the data from each patient into a pair of values to avoid alpha inflation due to the sample size when comparing conditions (morning vs afternoon; baseline vs. end of treatment). To this end, nine values from the morning assessment and the corresponding nine values at the afternoon administration ( 4 from phase A and 5 from phase B) were averaged. The effect size $(r)$ (i.e., the index measuring the magnitude of difference or change between two conditions, in this case baseline vs. end of the protocol and morning vs. afternoon) [30] was calculated as the $\mathrm{z}$ /square root $(\mathrm{N})$ (where $\mathrm{N}$ is the number of observations on which $\mathrm{z}$ is based) and will be hereafter formally referred to as not relevant $(r<0.1)$, small $(0.1<\mathrm{r}<0.3)$, medium $(0.3<\mathrm{r}<0.5)$, or large $(r>0.5)$ [31]. The Bonferroni correction for multiple testing was applied for a $p<0.005$ level of statistical significance on ten comparisons between morning and afternoon. The risk of misclassification of subjects in VS/UWS or MCS to the other clinical condition when relying only on a single (morning or afternoon) assessment was tested by the Odds Ratio and Risk Ratio in both subjects' groups.

\section{Results and discussion}

Individual CRS-r scores at the morning and afternoon scale administration are shown in Fig. 1. In both VS/ UWS and MCS subgroups and over the entire period of observation, the mean CRS-r global scores were higher at the morning assessment $(7 \pm 1.5$ and $11 \pm 1.9$ for VS/ UWS and MCS, respectively) than in the afternoon (6.3 \pm 1.3 and 10.1 \pm 1.9 ) (Wilcoxon's $\mathrm{z}=-3.916, p<0.0001$, $r=1.04$ and $\mathrm{z}=-5.195, p<0.0001, r=1.06$ ) (Fig. 2). Scores were higher in the morning in both Phases $\mathrm{A}$ and $\mathrm{B}$ in the MCS subgroup (Wilcoxon's $\mathrm{z}=-3.513$, $\mathrm{p}$ [correct $]<0.001, r=0.75$ and $\mathrm{z}=-3.843, \mathrm{p}$ [correct] $=0.018$, $r=0.82$, respectively), but only in Phase B the difference reached statistical significance in the VS/UWS subgroup (Wilcoxon's $\mathrm{z}=-3.840, \mathrm{p}$ [correct] $<0.001, r=1.02$ ). The morning vs. afternoon difference was observed also when considering posttraumatic subjects or patients with vascular brain injury separately (Wilcoxon's $\mathrm{z}=2.874$, $\mathrm{p}$ [correct $]=0.024, \quad r=0.67$ and Wilcoxon' $=\mathrm{s} \quad \mathrm{z}=-4.415$, $\mathrm{p}[$ correct $]=0.000, r=0.75$ respectively) (Fig. 1 ). It increased progressively over the 13 weeks. observation period in VS/UWS, while reaching a maximum between the $6^{\text {th }}$ and $9^{\text {th }}$ weeks of protocol to decline thereafter in the MCS subgroup (Figs. 2 and 3); as a result, the mean CRS-r global scores were higher at the end of the protocol compared to baseline in the VS/UWS $(7.6 \pm 2.4$ and $5.9 \pm$ 0.8 , respectively; morning-afternoon average; Wilcoxon's $\mathrm{z}:-2.347, \mathrm{p}=0.019$, effect size: $r=0.63$ ), but not in MCS (Figs. 2 and 3). The morning vs. afternoon difference in 


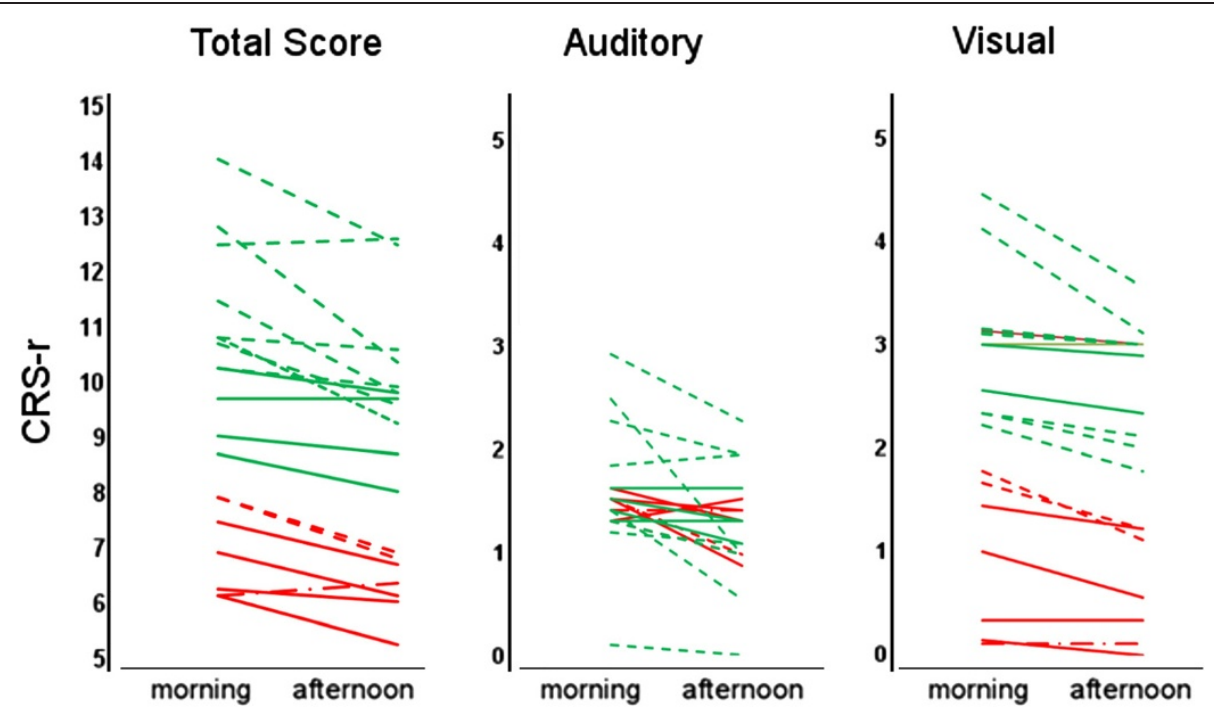

Fig. 1 CRS-r total and visual and auditory scores (means of 9 values at the morning and 9 at the afternoon assessments) for each VS/UWS (red) or MCS (green) subject. Dashed: vascular; continous: traumatic; dash-line: other etiologies

global score was accounted for by the visual and auditory items (Fig. 1) both in the MCS (Wilcoxon's $\mathrm{z}=-3.062, \mathrm{p}$ correct $=0.01, r=0.65$ and $\mathrm{z}=-3.721, \mathrm{p}$ correct $<0.001, r=0.79$ ) and VS/UWS subjects' groups (Wilcoxon's $\mathrm{z}=-2.874, \mathrm{p}$ correct $=0.02, r=0.77$ and $\mathrm{z}$ $=-2.744, \mathrm{p}$ correct $=0.03, \quad r=0.73$ ). No significant contributions from the motor, oromotor/verbal, communication and arousal subscales to the differences in the CRS-r global score were observed.

The measure of association in VS/UWS subjects between the CRS-r scores at the morning or afternoon assessments and the risk of wrong attribution to the MCS condition was significant (odds ratio $=4.75$; $95 \%$ confidence interval: $1.73-12.7 ; p=0.001$; relative risk $=1.4$; $95 \%$ coefficient interval: $1.15-1.7 ; p=0.001)$. The risk was higher in the phases $B$ (odds ratio $=5.09 ; 95 \%$ confidence interval: $1.38-18.7 ; p=0.014$; relative risk $=1.76$; $95 \%$ coefficient interval: $1.30-1.19 ; p=0.014$ ) than in phases A (odds ratio $=1.70 ; 95 \%$ confidence interval: 10.51-2.55; $p=0.5$; relative risk $=0.78 ; 95 \%$ coefficient interval: 2.04-1.86; $p=0.014)$. The risk of wrong classification was lower in MCS group (odds ratio $=0.49 ; 95 \%$ confidence interval: $0.087-2.73 ; p=0.44$; relative risk $=0.66$; $95 \%$ coefficient interval: $0.21-2.06 ; p=0.44)$. The estimated probability of observing during the rehabilitation protocol CRS-r items compatible with MCS in subjects diagnosed as VS/UWS was $30 \%$ at the morning (range: $0-55 \%$ ) and $9.5 \%$ at the afternoon assessments (range: 0-22\%).

These observations derive from a retrospective analysis of the CRS-r scores obtained during a conventional rehabilitation protocol that had not been designed to test differences in the subjects' responsiveness. In this respect, the differences between the protocol phases A and $B$ or the possible effects of intensive treatment are not suitable of detailed investigation or pathophysiological interpretation. Prospective studies are needed for this purpose. This caveat notwithstanding, the results both invite speculation and suggest caution in the use of the available clinical assessment tools. The CRS-r was administered at the same two time windows during the day when responsiveness had previously proved highest in chronic VS/UWS and MCS subjects [29]. The global score and visual and auditory subscores nevertheless proved higher in the morning than in the afternoon, with the mean differences in the CRS-r global score increasing with time in the VS/UWS subjects' subgroup; the morning vs. afternoon difference was about twice larger than the average improvement at the end of the protocol (54.2 \% vs. $28.8 \%)$ which confirms the large size effect estimated statistically. The variability of the CRS-r assessment was unrelated to etiology in our patients' sample, but a relationship with etiology cannot be ruled out in principle.

A positive visual pursuit response (a major CRS-r item) results of activation in the anterior and posterior midline structures of the brain (mesiofrontal and precuneal cortices) [32-35], which are metabolically impaired in the severe disorder of consciousness according to neuroimaging studies [36, 37]. Neuroimaging has also documented regional activation in response to stimulus conditions in VS/UWS [12, 14, 20, 38, 39]; responses to stimulus conditions purported to induce emotional reaction have been described [40-42]. Whether any of these responses may indicate "automatic" subcortical processing atypical for the VS/UWS or it marks higher order 

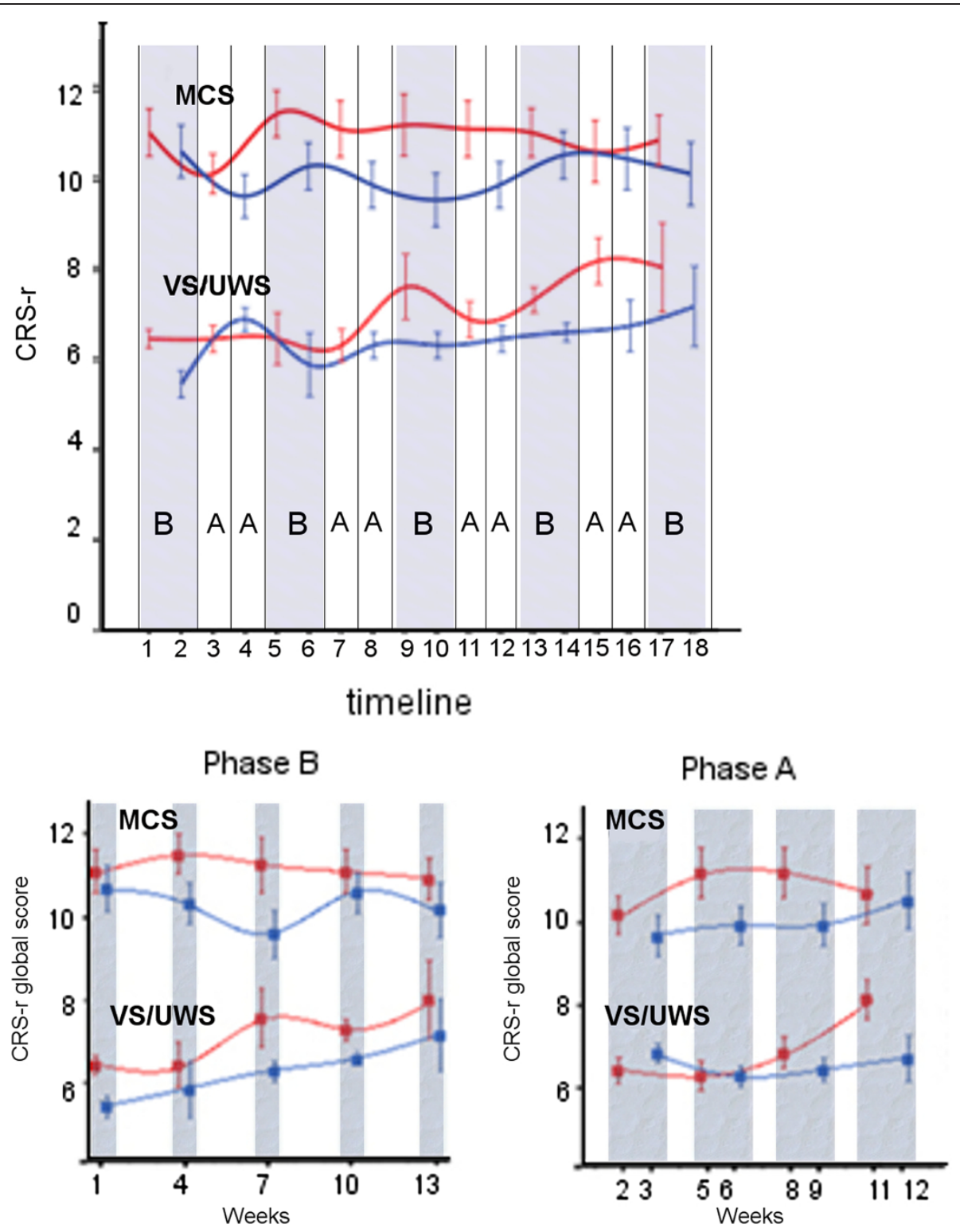

Fig. 2 Top: mean and SE across subjects of the CRS-r global score at the morning (red) and afternoon (blue) testing in VS/UWS and MCS. Bottom: CRS-r global scores (mean, SE) at the morning (red) and afternoon (blue) testing in the two treatment phases A and B

cortical activation and partially recovered consciousness, remains an unsolved controversy [19]. Yet, the observation of responses such as these is conventionally regarded as indicative of surviving modules of the corticocortical and brainstem-cortex functional interaction that is thought to sustain consciousness in the awake subject $[6,43-48]$ and is interfered with in the VS/UWS and MCS [2, 9, 10, 19, 21, 22, 36, 37, 39, 49, 50]. In this respect, the CRS-r reliability (as also documented by the relationship between EEG descriptors and CRS-r scores) [51] is not to be questioned on the ground of its variability during the day, nor is to be questioned the examiners' accuracy. Instead, the observed CRS-r differences between morning and afternoon are likely to reflect individual changes in the subject's level of visual, auditory and motor functioning conceivably due to changes in the neuronal/non-neuronal factors that modulate the brain state $[52,53]$. Spontaneous fluctuations of any of these factors may be expected to result in random differences in responsiveness rather than in systematic difference during the day; effects of fragmentary circadian/ultradian or otherwise cyclic processes (e.g., in metabolism) are thus also conceivable, albeit not documentable in the absence of multiple assessments at short intervals over the 24 h. period [22]. 


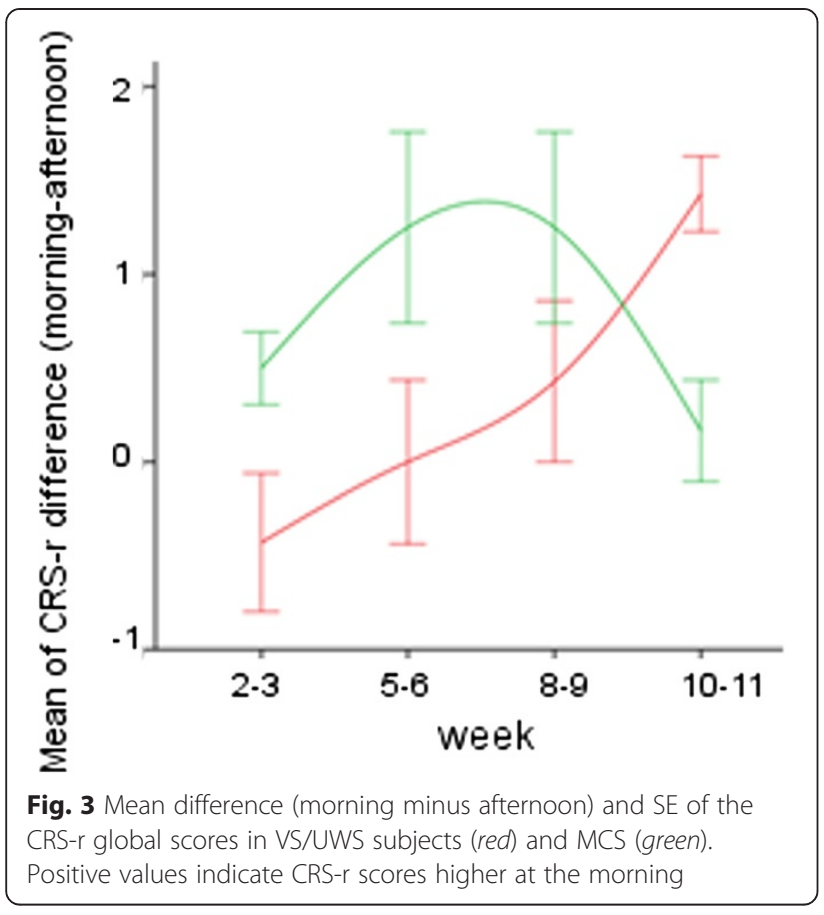

The extent to which the within-day variability in the CRS-r global, visual and auditory scores observed in this study may have clinical relevance in the diagnosis and early prognosis of subjects with disorder of consciousness remains to be studied in large patients' samples. Systematic investigation on the spontaneous variability over time of the relevant neuronal or non-neuronal parameters in the severe disorder of consciousness is also advisable and may help characterize these conditions in greater detail [52]. The possible source(s) of variability notwithstanding, the estimated risk of misclassification for a single random CRS-r testing appears compatible with the reported misdiagnosis between VS/UWS and $\operatorname{MCS}[3,4,6,7]$.

\section{Conclusions}

Multiple CRS-r assessments at different times of the day and monitoring over time are advisable. Diagnostic criteria taking also individual variability into a proper account may help reduce misdiagnosis between conditions sharing the underlying pathophysiology, but differing as to clinical picture, prognosis, required medical care and logistics, legal or popular perception of bioethical issues, allocated resources, healthcare policies, etc. [1, 6, 40, 54-56]. These considerations aside, the evidence of higher responsiveness in the morning compared to the afternoon invites discussion on whether the time window dedicated to treatment is irrelevant to rehabilitation or it should be planned accordingly.

\section{Abbreviations}

VS/UWS: Vegetative state/unresponsive wakefulness syndrome; MCS: Minimally conscious state; CRS-r: Coma recovery scale-revised; SE: Standard error.

\section{Competing interests}

The authors declare they have no financial or non-financial competing interests.

\section{Authors' contributions}

$M D, F R, F A$, and WGS, were involved in the conception and design of the study. FR performed the statistical analysis. MD, FR, FA, and WGS were involved in the analysis and interpretation of data. All authors were involved in the drafting and revision of the manuscript. All authors read and approved the final manuscript.

\section{Authors' information}

Not applicable.

\section{Author details}

${ }^{1}$ Institute S. Anna and RAN (Research in Advanced Rehabilitation), Crotone, Italy. ${ }^{2}$ Department of Neuroscience, Ophthalmology and Genetics, University of Genova, 3, Largo P. Daneo, 16132 Genova, Italy. ${ }^{3}$ Department of Psychiatry, State University of New York, Stony Brook, NY, USA.

Received: 28 April 2014 Accepted: 1 October 2015

Published online: 08 October 2015

\section{References}

1. Laureys S, Celesia GG, Cohadon F, Lavrijsen J, León-Carrión J, Sannita WG, et al. Unresponsive wakefulness syndrome: a new name for the vegetative state or apallic syndrome. BMC Med. 2010;8:68.

2. Giacino JT, Kalmar K. Diagnostic and prognostic guidelines for the vegetative and minimally conscious states. Neuropsychol Rehabil. 2005;15:166-74.

3. Andrews K, Murphy L, Munday R, Littlewood C. Misdiagnosis of the vegetative state: retrospective study in a rehabilitation unit. BMJ. 1996:313:13-6.

4. Bosco A, Lancioni GE, Belardinelli MO, Singh NN, O'Reilly MF, Sigafoos J. Vegetative state: efforts to curb misdiagnosis. Cogn Process. 2010;11:87-90.

5. Celesia GG, Sannita WG. Can patients in vegetative state experience pain and have conscious awareness? Neurology. 2013;80:328-9.

6. Dolce G, Sannita WG, European Task Force On The Vegetative State. The vegetative state: A syndrome seeking revision? Brain Inj. 2010;24:1628-9.

7. Schnakers C, Vanhaudenhuyse A, Giacino J, Ventura M, Boly M, Majerus S, et al. Diagnostic accuracy of the vegetative and minimally conscious state: clinical consensus versus standardized neurobehavioral assessment. BMC Neurol. 2009;9:35.

8. Bodart O, Laureys S, Gosseries O. Coma and disorders of consciousness: scientific advances and practical considerations for clinicians. Semin Neurol. 2013:33:083-90.

9. Giacino JT, Hirsch J, Schiff N, Laureys S. Functional neuroimaging applications for assessment and rehabilitation planning in patients with disorders of consciousness. Arch Phys Med Rehabil. 2006;87(12, Supplement):67-76. Neuroplasticity and Brain Imaging Research: Implications for Rehabilitation.

10. Laureys S, Lemaire C, Maquet P, Phillips C, Franck G. Cerebral metabolism during vegetative state and after recovery to consciousness. J Neurol Neurosurg Psychiatry. 1999;67:121-2.

11. Monti MM, Coleman MR, Owen AM. Neuroimaging and the vegetative state. Ann N Y Acad Sci. 2009;1157:81-9.

12. Owen AM, Coleman MR. Functional neuroimaging of the vegetative state. Nat Rev Neurosci. 2008;9:235-43.

13. Schiff ND, Rodriguez-Moreno D, Kamal A, Kim KHS, Giacino JT, Plum F, et al. fMRI reveals large-scale network activation in minimally conscious patients. Neurology. 2005;64:514-23.

14. Monti MM, Rosenberg M, Finoia P, Kamau E, Pickard JD, Owen AM. Thalamo-frontal connectivity mediates top-down cognitive functions in disorders of consciousness. Neurology. 2014; doi: 10.1212/ WNL.0000000000001123. 
15. Fernández-Espejo D, Bekinschtein T, Monti MM, Pickard JD, Junque C, Coleman MR, et al. Diffusion weighted imaging distinguishes the vegetative state from the minimally conscious state. Neurolmage. 2011;54:103-12.

16. Childs NL, Mercer WN, Childs HW. Accuracy of diagnosis of persistent vegetative state. Neurology. 1993;43:1465-5.

17. Majerus S, Gill-Thwaites H, Andrews K, Laureys S. Behavioral evaluation of consciousness in severe brain damage. In Progress in brain research. Volume 150. Edited by Laureys S. Elsevier: Liege (BL); 2005.397-413. The Boundaries of Consciousness: Neurobiology and Neuropathology.

18. Giacino JT, Kalmar K, Whyte J. The JFK coma recovery scale-revised: measurement characteristics and diagnostic utility. Arch Phys Med Rehabil. 2004;85:2020-9.

19. Giacino JT, Ashwal S, Childs N, Cranford R, Jennett B, Katz DI, et al. The minimally conscious state definition and diagnostic criteria. Neurology. 2002;58:349-53.

20. Bruno M-A, Vanhaudenhuyse A, Schnakers C, Boly M, Gosseries O, Demertz $A$, et al. Visual fixation in the vegetative state: an observational case series PET study. BMC Neurol. 2010;10:35.

21. Giacino JT. Disorders of consciousness: differential diagnosis and neuropathologic features. In: Seminars in neurology, vol. 17. New York: Thieme-Stratton Inc; 1997. p. 105-12.

22. Bruno M-A, Vanhaudenhuyse A, Thibaut A, Moonen G, Laureys S. From unresponsive wakefulness to minimally conscious PLUS and functional locked-in syndromes: recent advances in our understanding of disorders of consciousness. J Neurol. 2011;258:1373-84.

23. Dolce G, Quintieri M, Serra S, Lagani V, Pignolo L. Clinical signs and early prognosis in vegetative state: a decisional tree, data-mining study. Brain Inj. 2008;22:617-23.

24. Vanhaudenhuyse A, Schnakers C, Brédart S, Laureys S. Assessment of visual pursuit in post-comatose states: use a mirror. J Neurol Neurosurg Psychiatry. 2008;79:223-3.

25. Jennett B. The vegetative state. J Neurol Neurosurg Psychiatry. 2002;73:355-7.

26. Dolce G, Sazbon L. The post-traumatic vegetative state. Stuttgart: Thieme; 2002.

27. Dolce G, Lucca LF, Candelieri A, Rogano S, Pignolo L, Sannita WG. Visual pursuit in the severe disorder of consciousness. J Neurotrauma. 2011;28:1149-54.

28. Riganello F, Cortese MD, Dolce G, Sannita WG. Visual pursuit response in the severe disorder of consciousness: modulation by the central autonomic system and a predictive model. BMC Neurol. 2013;13:164

29. Candelieri A, Cortese MD, Dolce G, Riganello F, Sannita WG. Visual pursuit: within-Day variability in the severe disorder of consciousness.

J Neurotrauma. 2011;28:2013-7.

30. Rosenthal R. Effect sizes: Pearson's correlation, its display via the BESD, and alternative indices. Am Psychol. 1991;46:1086-7.

31. Hemphill JF. Interpreting the magnitudes of correlation coefficients. Am Psychol. 2003;58:78-9.

32. Krauzlis RJ. Neuronal activity in the rostral superior colliculus related to the initiation of pursuit and saccadic eye movements. J Neurosci. 2003;23:4333-44.

33. Krauzlis RJ, Lisberger SG. Temporal properties of visual motion signals for the initiation of smooth pursuit eye movements in monkeys. J Neurophysiol. 1994;72:150-62.

34. Newsome WT, Wurtz RH, Dursteler MR, Mikami A. Deficits in visual motion processing following ibotenic acid lesions of the middle temporal visual area of the macaque monkey. J Neurosci. 1985;5:825-40.

35. Tian JR, Lynch JC. Corticocortical input to the smooth and saccadic eye movement subregions of the frontal eye field in Cebus monkeys. J Neurophysiol. 1996;76:2754-71.

36. Laureys S, Perrin F, Brédart S. Self-consciousness in non-communicative patients. Conscious Cogn. 2007;16:722-41. Subjectivity and the Body.

37. Laureys S, Owen AM, Schiff ND. Brain function in coma, vegetative state, and related disorders. Lancet Neurol. 2004;3:537-46.

38. Cruse D, Chennu S, Chatelle C, Bekinschtein TA, Fernández-Espejo D, Pickard $\mathrm{JD}$, et al. Bedside detection of awareness in the vegetative state: a cohort study. Lancet. 2012;378:2088-94.

39. Monti MM, Laureys S, Owen AM. The vegetative state. BMJ. 2010;341:C3765-5

40. Dolce G, Riganello F, Quintieri M, Candelieri A, Conforti D. Personal interaction in the vegetative state: a data-mining study. J Psychophysiol. 2008;22:150-6.
41. Riganello F, Candelieri A, Quintieri M, Conforti D, Dolce G. Heart rate variability: An index of brain processing in vegetative state? an artificial intelligence, data mining study. Clin Neurophysiol. 2010;121:2024-34.

42. Machado C, Korein J, Aubert E, Bosch J, Alvarez MA, Rodríguez R, et al. Recognizing a Mother's voice in the persistent vegetative state. Clin EEG Neurosci. 2007;38:124-6.

43. Hassler R, Rolle Y, Streicher H. Pathophisiologie Der Bewustseintatigkeit. Stuttgart: Georg Thieme; 1972.

44. Boussaoud D, Ungerleider LG, Desimone R. Pathways for motion analysis: cortical connections of the medial superior temporal and fundus of the superior temporal visual areas in the macaque. J Comp Neurol. 1990;296:462-95.

45. Gottlieb JP, MacAvoy MG, Bruce CJ. Neural responses related to smoothpursuit eye movements and their correspondence with electrically elicited smooth eye movements in the primate frontal eye field. J Neurophysiol. 1994;72:1634-53.

46. Kim JS, Sharpe JA. The vertical vestibulo-ocular reflex, and its interaction with vision during active head motion: effects of aging. J Vestib Res. 2001;11:3-12.

47. Zeman A. Consciousness. In Encyclopedia of cognitive science. John Wiley \& Sons, Ltd; Chichester (UK); 2006.

48. Rosano C, Krisky CM, Welling JS, Eddy WF, Luna B, Thulborn KR, et al. Pursuit and saccadic Eye movement subregions in human frontal Eye field: a highresolution fMRI investigation. Cereb Cortex. 2002;12:107-15.

49. Gusnard DA, Raichle ME. Searching for a baseline: functional imaging and the resting human brain. Nat Rev Neurosci. 2001;2:685-94.

50. Taylor CM, Aird VH, Tate RL, Lammi MH. Sequence of recovery during the course of emergence from the minimally conscious state. Arch Phys Med Rehabil. 2007:88:521-5.

51. Lechinger J, Bothe K, Pichler G, Michitsch G, Donis J, Klimesch W, et al. CRS$\mathrm{R}$ score in disorders of consciousness is strongly related to spectral EEG at rest. J Neurol. 2013;260:2348-56.

52. Sannita WG. Individual variability, end-point effects and possible biases in electrophysiological research. Clin Neurophysiol. 2006;117:2569-83.

53. Cruse D, Thibaut A, Demertzi A, Nantes JC, Bruno M-A, Gosseries O, et al. Actigraphy assessments of circadian sleep-wake cycles in the vegetative and minimally conscious states. BMC Med. 2013;11:18.

54. Racine E, Amaram R, Seidler M, Karczewska M, Illes J. Media coverage of the persistent vegetative state and end-of-life decision-making. Neurology. 2008;71:1027-32

55. Katz-Leurer $\mathrm{M}$, Rotem $\mathrm{H}$, Keren $\mathrm{O}$, Meyer $\mathrm{S}$. Heart rate and heart rate variability at rest and during exercise in boys who suffered a severe traumatic brain injury and typically-developed controls. Brain Inj. 2010:24:110-4.

56. Luauté J, Maucort-Boulch D, Tell L, Quelard F, Sarraf T, Iwaz J, et al. Longterm outcomes of chronic minimally conscious and vegetative states. Neurology. 2010;75:246-52.

\section{Submit your next manuscript to BioMed Central and take full advantage of:}

- Convenient online submission

- Thorough peer review

- No space constraints or color figure charges

- Immediate publication on acceptance

- Inclusion in PubMed, CAS, Scopus and Google Scholar

- Research which is freely available for redistribution 